\section{PTH-45 USING INFORMATION VIDEOS TO IMPROVE PATIENT SATISFACTION IN ENDOSCOPY (INFOVIPE)}

Ephraim Broder*, Laith Al-Rubaiy, Rayhan Chaudhry, Stephen Preston, Adam Humphries. Imperial College, London, UK

\subsection{6/gutjpl-2021-BSG.360}

Introduction Endoscopy is a rapidly developing discipline with new techniques and procedures being introduced each year. Patient-Reported Outcome Measures are reputable tools that may address the increasing need to evaluate how evolving endoscopy practice affects patient satisfaction. The consenting process is central to patient perception; using information videos as additional tools to aid consent and improve the quality of care in endoscopy is not well established. The aim of this project was to develop, implement and validate the use of patient educational videos to improve patients' satisfaction and experience in endoscopy.

Methods This was a prospective service quality improvement study. Eligible patients were invited to watch the educational videos in addition to standard practice. We compared this group with a matched cohort of patients who were receiving standard care of postal information leaflets. Patient satisfaction was measured through the validated Gastrointestinal Endoscopy Satisfaction Questionnaire (GESQ).

Results The total average GESQ score was higher in the intervention group compared to the control group, but the difference was not statistically significant $(p=0.397)$. A sub-score was calculated from the four questions relating to pre-procedural information. The average sub-score was significantly higher in the video group $(p=0.038)$. The video group had a lower cancellation rate (4\%) compared to the control group (20\%).

Conclusions The videos were well-received and patients found them useful and easy to understand. Further research is still needed to determine if they reduce patient anxiety. Meanwhile, it appears to be appropriate to implement these videos into routine practice while constantly monitoring patient feedback, updating them as necessary. Eventually, these videos could be rolled out across the UK as a standard part of the information package prior to endoscopy.

\section{PTH-46 TELEMEDICINE OUTPATIENT HEPATOLOGY CLINICS: A PATIENT SURVEY AND NOTES AUDIT DURING THE COVID-19 PANDEMIC}

Michelle Camarata*, Claudia Moore-Gillon*, Aaliya Gilbert*, Belinda Smith, Ameet Dhar. St Marys Hospital, Imperial College London, London, UK

\subsection{6/gutjnl-2021-BSG.361}

Introduction The Covid pandemic in March 2020 necessitated the introduction of virtual clinic phone reviews (VC) as an alternative to face-to-face reviews (F2F). We aimed to study patient satisfaction and clinical effectiveness of $\mathrm{VC}$ in the setting of general hepatology outpatients.

Methods We conducted a phone survey of patients $(n=60)$ who underwent a VC during a week in November 2021 from five general hepatology clinics from different consultants at a tertiary referral centre in London. Decompensated liver disease patients from specialist alcohol and cirrhosis clinics were excluded. Patient satisfaction was scored from $1-10(10=$ extremely satisfied). We also performed a retrospective notes audit to review clinician rated effectiveness of the clinics looking at criteria such as appropriate triage, whether investigations/bloods were required to complete the review or if a physical exam was needed.

Results The survey was completed by 39 of 60 patients contacted (median age 54 yrs, range 23-85 yrs; M:F 19: 19 male, 4 had cirrhosis). 32 clinic visits were follow up and 7 were new. $90 \%$ of patients had access to internet and 100\% had a phone. Viral hepatitis was the most common aetiology (42\%), then NAFLD (13\%) and AIH (10\%). The median satisfaction score was 8 (4.5-10). Compared to F2F, $72 \%$ reported they had enough time, $85 \%$ felt the doctor listened, $85 \%$ got answers to questions, 90\% had confidence in their doctor and $95 \%$ understood the management plan. $63 \%$ would prefer the option of VC post pandemic. There was no significant difference in ages between patients that preferred $\mathrm{VC}$ and those that didn't (mean $51 \mathrm{y}$ vs $55 \mathrm{y} ; \mathrm{p}=0.22$ ). Travel distance and first language also did not appear to be associated with preference for VC. There was a significant difference $(p=0.03)$ in unemployment between patients that preferred F2F (71\%) compared to VC (29\%). Most patients that preferred future VC still stated their ideal ratio would be 50:50. VC were rated as ineffective if requested bloods tests were not done within 4 weeks $(42 \%, n=14)$ or a physical exam would have been required $(n=2)$.

Conclusion Our results indicate that the majority of patients with well compensated liver disease found virtual reviews acceptable but would still prefer an equal ratio of $\mathrm{VC}$ and F2F appointments in the future. Patient preference was not predicted by age, travel distance or first language, in contrast to employment status. Further studies are required to improve the clinical effectiveness of virtual reviews and patient satisfaction, including optimising triage criteria and improving access to investigations.

2910 characters ( $\max 3000$ inc. spaces - includes title, authors and affiliations)

\section{PTH-47 MANAGEMENT OF BLEEDING RISK IN LIVER DISEASE PATIENTS UNDERGOING INVASIVE PROCEDURES AT GLOUCESTERSHIRE HOSPITALS}

Pedram Modarres*, R Frewin, B Odedra, F Cackett, M Popli, C Hollywood. Gloucestershire Hospitals NHSFT, UK

\subsection{6/gutjnl-2021-BSG.362}

Introduction Liver disease patients undergo 'rebalanced haemostasis' whereby reduction in endogenous coagulation factors is offset by prothrombotic counter-effects. Targets of platelets $>50 \mathrm{x} \times 10^{9} / \mathrm{L}$ and fibrinogen $>1.2 \mathrm{~g} / \mathrm{l}$ and use of vitamin $\mathrm{K}$ may prevent bleeding in high-risk procedures. PT/INR do not equate to bleeding risk and fresh frozen plasma (FFP) is not recommended as prophylaxis in liver disease.[1] Ascitic drain $\&$ liver biopsy $(\mathrm{AD} / \mathrm{LB})$ are commonly performed invasive procedures with associated haemorrhagic complications.

Aims 1) Assess recent practice of the management of bleeding risk in liver disease patients undergoing $\mathrm{AD} / \mathrm{LB}$. 2) Develop a multi-speciality trust proforma on managing bleeding risk in liver disease patients undergoing $\mathrm{AD} / \mathrm{LB}$ based on national guidelines.[1]

Methodology A retrospective analysis of medical notes from August 2017-19 at Gloucestershire Hospitals was performed. Inclusion criteria were inpatient $\mathrm{AD} / \mathrm{LB}$ performed on liver cirrhosis patients; and platelets $\leq 50 \times 10^{9} / \mathrm{L}$, fibrinogen $\leq 1.2 \mathrm{~g} / \mathrm{l}$, 